\title{
Regulation of Adrenal Aldosterone Production by Serine Protease Prostasin
}

\author{
Takehiro Ko, ${ }^{1}$ Yutaka Kakizoe, ${ }^{1}$ Naoki Wakida, ${ }^{1}$ Manabu Hayata, ${ }^{1}$ Kohei Uchimura, ${ }^{1}$ \\ Naoki Shiraishi, ${ }^{1}$ Taku Miyoshi, ${ }^{1}$ Masataka Adachi, ${ }^{1}$ Shizuka Aritomi, ${ }^{2}$ Tomoyuki Konda, \\ Kimio Tomita, ${ }^{1}$ and Kenichiro Kitamura ${ }^{1}$ \\ ${ }^{1}$ Department of Nephrology, Kumamoto University Graduate School of Medical Sciences, 1-1-1 Honjo, Kumamoto,
Kumamoto 860-8556, Japan
${ }^{2}$ Pharmaceutical Research Laboratories, Ajinomoto Co., Inc., 1-1 Suzuki-cho, Kawasaki, Kawasaki 210-8681, Japan
}

Correspondence should be addressed to Kenichiro Kitamura, ken@gpo.kumamoto-u.ac.jp

Received 25 September 2009; Revised 11 November 2009; Accepted 23 November 2009

Academic Editor: Karl Chai

Copyright ( 92010 Takehiro Ko et al. This is an open access article distributed under the Creative Commons Attribution License, which permits unrestricted use, distribution, and reproduction in any medium, provided the original work is properly cited.

\begin{abstract}
A serine protease prostasin has been demonstrated to have a pivotal role in the activation of the epithelial sodium channel. Systemic administration of adenovirus carrying human prostasin gene in rats resulted in an increase in plasma prostasin and aldosterone levels. However, the mechanism by which the elevation of prostasin levels in the systemic circulation stimulated the plasma aldosterone levels remains unknown. Therefore, we examined if prostasin increases the aldosterone synthesis in a human adrenocortical cell line (H295R cells). Luciferase assay using CYP11B2 promoter revealed that prostasin significantly increased the transcriptional activity of CYP11B2. Prostasin significantly increased both CYP11B2 mRNA expression and aldosterone production in a dose-dependent manner. Surprisingly, treatment with camostat mesilate, a potent prostasin inhibitor, had no effect on the aldosterone synthesis by prostasin and also a protease-dead mutant of prostasin significantly stimulated the aldosterone production. A T-type/L-type calcium channel blocker and a protein kinase C (PKC) inhibitor significantly reduced the aldosterone synthesis by prostasin. Our findings suggest a stimulatory effect of prostasin on the aldosterone synthesis by adrenal gland through the nonproteolytic action and indicate a new role of prostasin in the systemic circulation.
\end{abstract}

\section{Introduction}

Proteases are involved in numerous essential biological processes including blood clotting, controlled cell death, and tissue differentiation. Prostasin is a glycosylphosphatidylinositol-(GPI-)anchored and/or secreted serine protease purified from human seminal fluid [1] and is expressed in the kidney, prostate, liver, lung, pancreas, and colon [2]. Prostasin has been identified as a potential regulator of the epithelial sodium channel function in the kidney, lung, and airways. Previously we showed that aldosterone increased prostasin abundance in rat and human [3] and also demonstrated a positive relationship between urinary prostasin levels and plasma or urinary aldosterone levels in human [4]. GPI-anchor free form of prostasin is also found in serum, but the physiological role of prostasin, especially in the systemic circulation, remains largely undetermined. In 2003, Wang et al. demonstrated that systemic administration of adenovirus carrying human prostasin gene in rats resulted in an elevation of plasma prostasin levels, plasma aldosterone concentration, and blood pressure [5]. However, the mechanism by which prostasin increases plasma aldosterone levels remains to be elucidated.

The adrenal cortex is the site of synthesis of the potent mineralocorticoid, aldosterone, and the glucocorticoid, cortisol. Aldosterone synthase (CYP11B2), which is expressed only within the zona glomerulosa of the adrenal cortex, is responsible for converting deoxycorticosterone to aldosterone [6-9]. The two most important physiological stimuli of aldosterone secretion, angiotensin II and extracellular $\mathrm{K}^{+}$ [10], exert their effects through generating cytoplasmic $\mathrm{Ca}^{2+}$ signal. Meanwhile, proteolytic enzymes such as trypsin have 
also been demonstrated to stimulate aldosterone secretion directly from intact rat adrenal capsular tissue [11-13]. Although the molecular mechanisms by which trypsin activates aldosterone production in adrenal cortex remain to be clarified, a possible involvement of protease-activated receptor-1 (PAR-1) has been suggested [14].

Therefore, in the current studies we hypothesized that serine protease prostasin increases aldosterone production by the adrenal gland and investigated the effect of prostasin on CYP11B2 expression and aldosterone production in H295R cells. We also examined a possible molecular mechanism by which prostasin activates aldosterone production.

\section{Methods}

2.1. Reagents. A protein kinase C inhibitor, Ro-31-8220, was purchased from Sigma-Aldrich (St Louis, MO, USA). Camostat mesilate was kindly supplied by Ono Pharmaceutical Co., Ltd. (Osaka, Japan). Efonidipine hydrochloride was from Shionogi \& Co., Ltd. (Osaka, Japan), and Valsartan was from Novartis Pharma K.K. (Tokyo, Japan).

\subsection{Purification of Wild Type and Protease-Dead Recombinant} Human Prostasin. Wild type recombinant human prostasin was prepared as described previously [15]. Briefly, a cDNA for recombinant human prostasin was created by inserting an enterokinase cleavage site, Asp-Asp-Asp-Asp-Lys, between the light chain and heavy chain and by replacing the $\mathrm{C}$ terminal membrane anchoring domain with a $6 \times$ His tag. To generate protease-dead mutant prostasin, catalytic triad of prostasin, His85, Asp134, and Ser238 were all replaced by Ala using QuikChange Site-Directed Mutagenesis Kit (Stratagene, La Jolla, CA, USA). Recombinant baculovirus carrying these cDNAs were developed and silkworm larvae were infected with these virus. After infection, hemolymph was collected and recombinant prostasin was purified by using a Ni-sepharose column (HisTrap HP: GE Healthcare Bio-Sciences, Piscataway, NJ, USA), an ion exchange column (HiTrap Q: GE Healthcare Bio-Sciences), and a gel filtration column (Superdex 200 10/300 GL: GE Healthcare BioSciences). Purified recombinant prostasin was treated with enterokinase (EK Max: Invitrogen, Carlsbad, CA, USA) at $37^{\circ} \mathrm{C}$ to generate mature recombinant prostasin by cleaving the enterokinase cleavage site. The enterokinase was removed from the reaction mixture by enterokinase removal kit (Sigma-Aldrich). Proteolytic activity of prostasin was determined by the enzymatic assay as described previously [4].

2.3. Cell Culture. The H295R human adrenocortical cell line was purchased from the American Type Culture Collection (Manassas, VA, USA) and cultured in DMEM/F12 medium (Gibco Invitrogen, Burlington, ON, Canada) containing 2.5\% NuSerum (BD Biosciences, Bedford, MA, USA), 1\% ITS (BD Biosciences). The cells were maintained at $37^{\circ} \mathrm{C}$ under a humid atmosphere of $95 \%$ air/5\% $\mathrm{CO}_{2}$. Briefly, 3 $\times 10^{5}$ cells were plated onto 12 -well plates and incubated until $80 \%$ confluent, and then medium was replaced with fresh medium containing $0.2 \% \mathrm{NuSerum}$ and the cells were incubated for an additional 24 hours. This medium was then replaced with medium containing the recombinant prostasin with or without different agents. Cells were cultured for an additional 3, 6, 12, 24 hours for RNA isolation or 48 hours for aldosterone assay in the culture media.

2.4. Luciferase Reporter Assay. The human CYP11B2 promoter-luciferase construct (pB2-1521) used in the current study was kindly provided by Bassett et al. (University of Texas Southwestern Medical Center, Dallas, Texas, USA) [16]. H295R cells $\left(4.5 \times 10^{5}\right.$ cells/well $)$ were seeded onto 12 -well plate and grown to reach $50 \%$ confluency. FuGENE6 transfection reagent (Roche Applied Science, Mannheim, Germany) was used to cotransfect $0.3 \mathrm{pmol}$ of pB2-1521 and $3.8 \mathrm{fmol}$ of pRL-TK (Promega, Madison, WI, USA) into the cells. For the determination of reporter activity, firefly luciferase activity from the pB21521 and Renilla luciferase activity from the pRL-TK were measured by the Dual Luciferase Assay System (Promega) on a luminometer (model TD-20/20, Turner Designs Inc., Sunnyvale, CA, USA).

2.5. Aldosterone Assay. Aldosterone contents in the culture media were determined by Aldosterone Express EIA Kit (Cayman chemical, Ann Arbor, MI, USA) according to the manufacturer's instruction.

2.6. Real-Time PCR Analysis. Total RNA was extracted from H295R cells by using RNeasy Mini Kit (Qiagen, Hilden, Germany). Five hundred nanograms of total RNA was reverse-transcribed to cDNA with QuantiTect Reverse Transcription Kit (Qiagen). TaqMan probes for human CYP11B2, PAR-1, PAR-2, and GAPDH were all purchased from Applied Biosystems (Foster city, CA, USA). Realtime PCR was performed with LightCycler 480 Real-Time PCR System (Roche Applied Science). Statistical analysis of results was performed with the $\Delta \mathrm{Ct}$ value ( $\mathrm{Ct}_{\text {gene of interest }}{ }^{-}$ $\left.\mathrm{Ct}_{\mathrm{GAPDH}}\right)$. Relative gene expression was obtained using the $\Delta \Delta \mathrm{Ct}$ method $\left(\mathrm{Ct}_{\text {sample }}-\mathrm{Ct}_{\text {calibrator }}\right)$.

2.7. Adenovirus Construction and Infection. Adenovirus vector, Ad-hPRSS8, in which the expression of human prostasin cDNA was under the control of the cytomegalovirus (CMV) promoter/enhancer was constructed and prepared by using Transpose-Ad system (Qbiogene, Illkirch, France). Adenovirus harboring LacZ gene driven by CMV promoter/enhancer (Ad-LacZ) was used as control. All viruses were amplified in transcomplemental 293 cells and purified by cesium chloride gradient ultracentrifugation. H295R cells $\left(3 \times 10^{5}\right.$ cells/well $)$ were seeded onto 12 -well plate in DMEM/F12 medium containing 2.5\% UltroserSF (Biosepra, Cergy St. Christophe, France), and 1\% ITS. Three days after seeding, cells were infected with $6 \times 10^{5} \mathrm{pfu} /$ well of adenovirus in serum-free DMEM/F12 for 1 hour, and then the medium was replaced with DMEM/F12 containing $0.2 \%$ NuSerum. Forty eight hours after infection, the culture 
medium was collected for the detection of prostasin expression and the cells were harvested for the evaluation of the CYP11B2 mRNA expression.

2.8. Application of siRNA. H295R cells were transfected with human PAR-1 siRNA (Silencer Select Validated siRNA, ID: s4923, Ambion Inc., Austin, TX, USA) or human PAR-2 siRNA (ID: s4926, Ambion) or control siRNA (ID: 4390843, Ambion) by using Lipofectamine RNAiMAX (Invitrogen) according to the manufacture's instruction. Forty-eight hours after transfection, the cells were treated with recombinant prostasin in DMEM/F12 with $0.2 \%$ NuSerum for 24 hours and then harvested for the total RNA isolation.

2.9. Protein Preparation and Immunoblottings. Forty-eight hours after adenovirus infection, $200 \mu \mathrm{L}$ of culture medium was concentrated and electrophoresed on 12\% SDSpolyacrylamide gels. The blots were probed with a monoclonal antibody against prostasin (BD Biosciences) as described previously [17]. Bands were visualized by chemiluminescence substrate (ECL; Amersham Pharmacia Biotech, Buckinghamshire, UK).

2.10. Statistical Analysis. Statistical significance was evaluated using the two-tailed, paired Student's $t$-test for comparisons between two means, or ANOVA analysis followed by the Newman-Keuls method for more than two means. A value of $P<.05$ was regarded as statistically significant. Results are reported as mean \pm SD.

\section{Results}

3.1. Effect of Prostasin on CYP11B2 Expression and Aldosterone Production. To examine the effect of prostasin on the transcriptional activity of CYP11B2 gene, H295R cells were transfected with pB2-1521 and treated with recombinant prostasin for 24 hours. As shown in Figure 1(a), $400 \mu \mathrm{g} / \mathrm{mL}$ of prostasin significantly enhanced the transcriptional activity of CYP11B2 by approximately 1.6 -fold. Next, we determined the endogenous mRNA expression of CYP11B2 in H295R cells following 24-hour treatment with various concentrations of prostasin. Prostasin significantly augmented the CYP11B2 expression in a dose-dependent manner within a range from 100 to $400 \mu \mathrm{g} / \mathrm{mL}$ (Figure 1(b)). We also studied the time-course of the prostasin-mediated increase in CYP11B2 mRNA. Cells were treated with $100 \mu \mathrm{g} / \mathrm{mL}$ of prostasin for 3, 6, and 24 hours, and the expression of CYP11B2 was evaluated by the real-time PCR. Treatment with prostasin significantly induced CYP11B2 expression from 6 to 24 hours (Figure 1(c)). Furthermore, aldosterone contents in the culture medium were determined following 48 -hour incubation with $100 \mu \mathrm{g} / \mathrm{mL}$ or $400 \mu \mathrm{g} / \mathrm{mL}$ of prostasin. As shown in Figure 1(d), prostasin dosedependently stimulated aldosterone production in H295R cells.

3.2. Adenovirus-Mediated Gene-Transfer of Prostasin in H295R Cells Markedly Enhanced the Expression of CYP11B2 $m R N A$. To confirm the effect of prostasin on CYP11B2 expression, we infected H295R cells, which produce little endogenous prostasin protein, with adenovirus carrying human prostasin cDNA (Ad-hPRSS8) or control virus carrying LacZ cDNA (Ad-LacZ). The cells infected with AdhPRSS8 showed significantly higher mRNA expression of CYP11B2 and aldosterone production by 3.5-fold and 2.1fold, respectively, than the cells with Ad-LacZ (Figures 2(a) and 2(b)). The increase in CYP11B2 expression and aldosterone production were comparable between adenovirus infection and recombinant protein treatment although significantly higher amount of prostasin was applied to the cells when treated with recombinant prostasin. Probably this may be due to the differences in the quality and biological efficacy between the prostasin protein produced by the adenovirusmammalian cell system and by the baculovirus-insect cell system.

3.3. Effect of Protease-Activated Receptors (PARs) Gene Silencing on Prostasin-Induced CYP11B2 Expression in H295R Cells. Since trypsin has been demonstrated to stimulate aldosterone production through the activation of PAR-1 in rat adrenal cortex [11-14, 18], we examined if PAR is involved in the upregulation of CYP11B2 expression by prostasin. Because we detected both PAR-1 and PAR-2 expression in H295R cells (data not shown), we knocked down the expression of PAR-1 or PAR-2 by using specific siRNA and treated the cells with $400 \mu \mathrm{g} / \mathrm{mL}$ of prostasin for 24 hours. The knock down efficiency was approximately $90 \%$ at the mRNA levels (data not shown). As shown in Figure 2(c), the gene silencing of either PAR-1 or PAR-2 did not abolish the prostasin-induced CYP11B2 expression, suggesting that PAR signaling is not involved in the prostasin-mediated aldosterone synthesis.

3.4. Proteolytic Activity Is Not Required for the ProstasinInduced CYP11B2 Expression and Aldosterone Production in H295R Cells. To determine if the proteolytic activity of prostasin is required for the upregulation of aldosterone synthesis, we treated H295R cells with prostasin $(400 \mu \mathrm{g} / \mathrm{mL})$ in the presence or absence of camostat mesilate $(100 \mu \mathrm{M})$ which is a potent prostasin inhibitor in vitro $[15,19]$. Camostat mesilate $(100 \mu \mathrm{M})$ almost completely inhibited prostasin activity $(400 \mu \mathrm{g} / \mathrm{mL})$ in vitro by $98.3 \pm 0.8 \%$. Figures 3(a) and 3(b) showed that camostat mesilate had no effect on CYP11B2 expression and aldosterone production. To confirm these observations, we generated a proteasedead mutant of recombinant prostasin and studied the effect of mutant prostasin on the aldosterone synthesis. To our surprise, mutant prostasin $(400 \mu \mathrm{g} / \mathrm{mL})$ substantially increased CYP11B2 expression and aldosterone production to the same extent as wild type prostasin (Figures 3(c) and $3(d))$.

3.5. Effect of Angiotensin II Type 1 Receptor Blocker (ARB), Calcium Channel Blocker (CCB), or Protein Kinase C Inhibitor on Prostasin-Induced CYP11B2 Expression and Aldosterone Production in H295R Cells. Next, we examined the effect of 


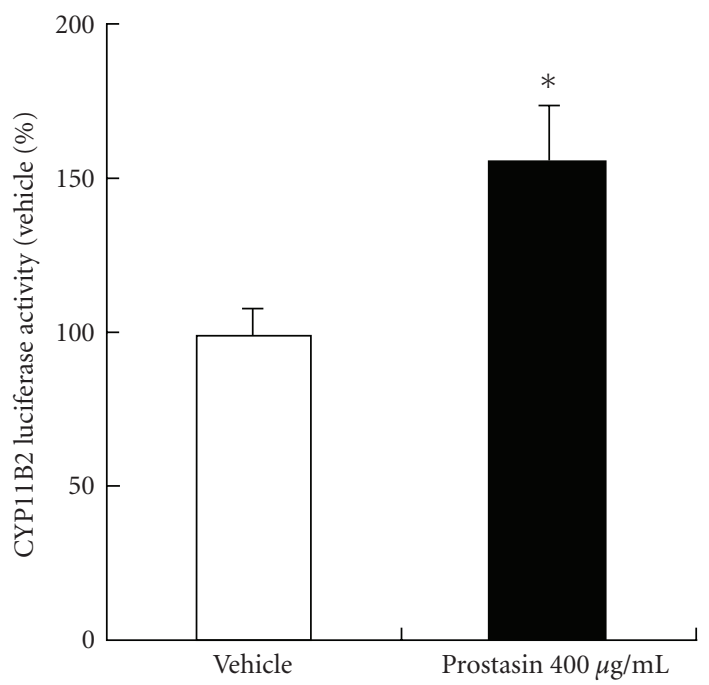

(a)

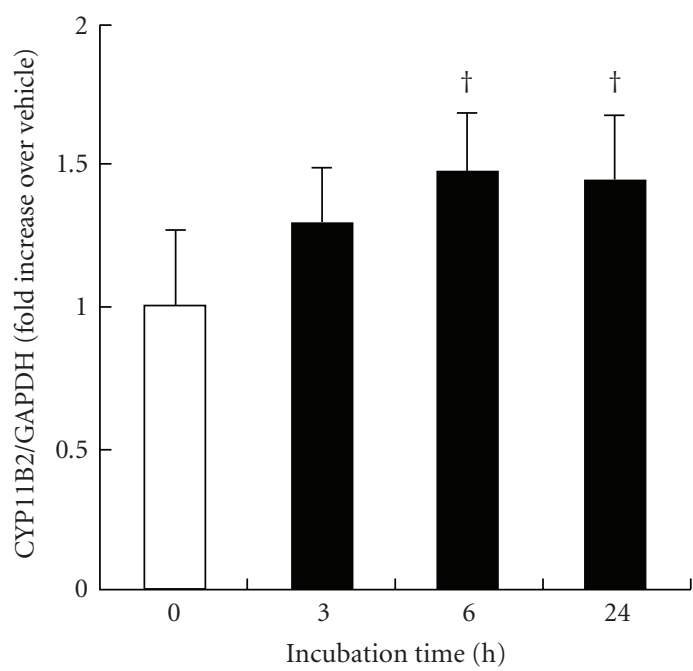

(c)

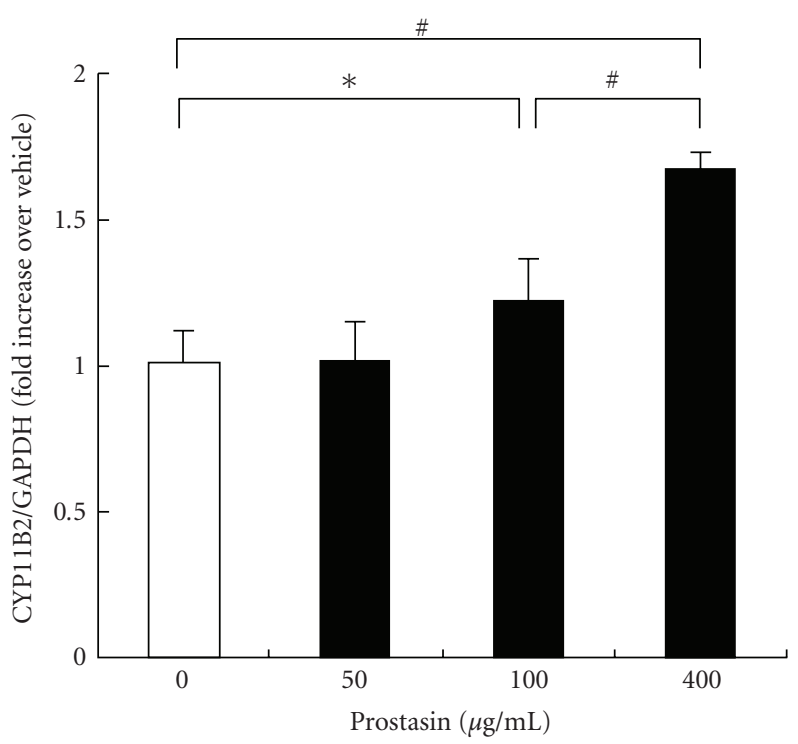

(b)

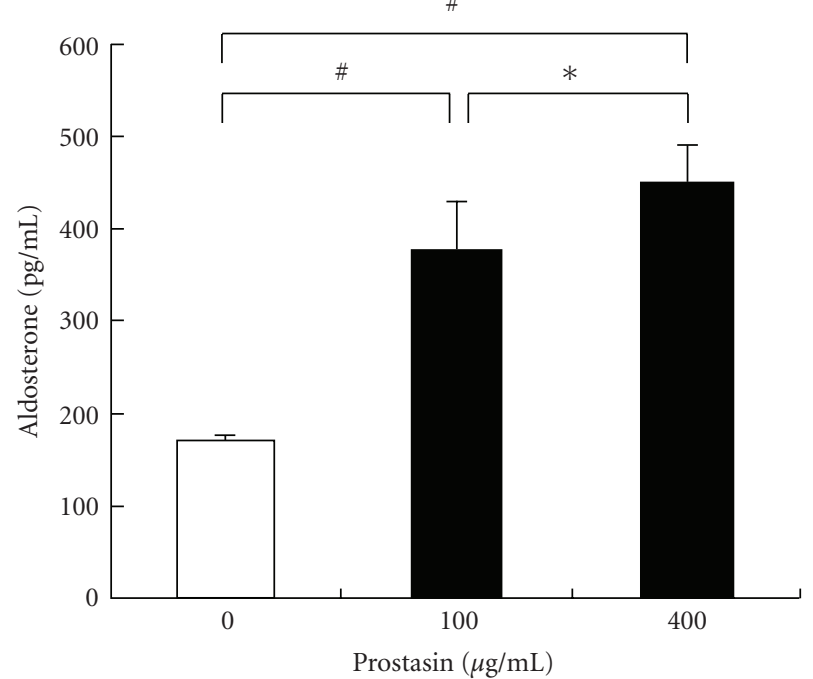

(d)

FIgURE 1: (a) Effect of prostasin on human CYP11B2 promoter activity in H295R cells. Cells were transfected with human CYP11B2 promoter-luciferase construct (pB2-1521) for 24 hours and then treated with vehicle or prostasin for another 24 hours. (b) Dose-dependent effect of prostasin on CYP11B2 mRNA expression in H295R cells. Cells were treated with increasing doses of prostasin for 24 hours. (c) Time course of CYP11B2 mRNA induction by prostasin in H295R cells. Cells were treated with $100^{-} \mathrm{g} / \mathrm{mL}$ of prostasin for $0,3,6$, and 24 hours. (d) Dose-dependent effect of prostasin on aldosterone production in H295R cells. Cells were treated with increasing concentrations of prostasin for 48 hours. Values are means $\pm \mathrm{SD}(n=6) .{ }^{*} P<.05,{ }^{\dagger} P<.01$, and ${ }^{\#} P<.001$.

$\mathrm{ARB}, \mathrm{CCB}$, or protein kinase $\mathrm{C}$ inhibitor on the aldosterone synthesis by prostasin in H295R cells. Cells were treated with prostasin $(400 \mu \mathrm{g} / \mathrm{mL})$ for 24 hours in the presence or absence of valsartan (100 nM), efonidipine (100 nM), or a protein kinase $\mathrm{C} \varepsilon$ inhibitor Ro-31-8220 (1 $\mu \mathrm{M})$. Valsartan had no effect on the prostasin-induced CYP11B2 expression and aldosterone production, while efonidipine significantly reduced both (Figures $4(\mathrm{a})$ and $4(\mathrm{~b})$ ). Treatment with Ro-318220 reduced the CYP11B2 mRNA expression to the control levels and significantly inhibited the aldosterone production (Figures 4(c) and 4(d)).

\section{Discussion}

In the current studies, we demonstrated the following findings: (1) prostasin increased the transcriptional activity of CYP11B2 promoter in H295R cells, (2) prostasin increased both CYP11B2 mRNA expression and aldosterone production in $\mathrm{H} 295 \mathrm{R}$ cells in a time and dosedependent manner, (3) augmentation of both CYP11B2 mRNA expression and aldosterone production was independent of the proteolytic activity of prostasin, and (4) induction of CYP11B2 expression and aldosterone production by 


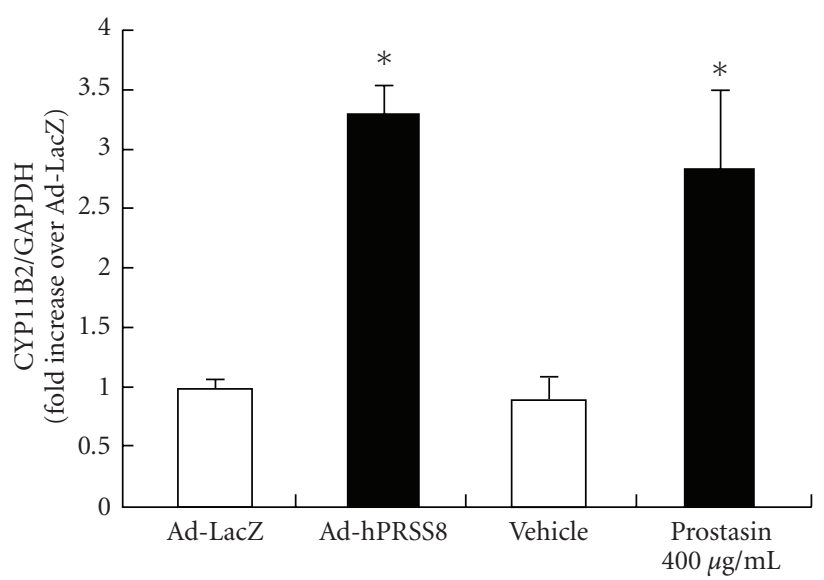

(a)

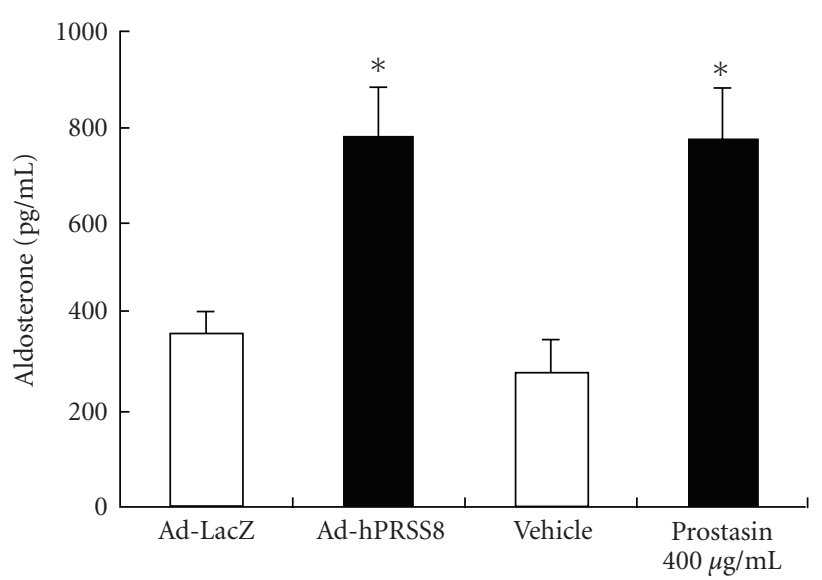

(b)

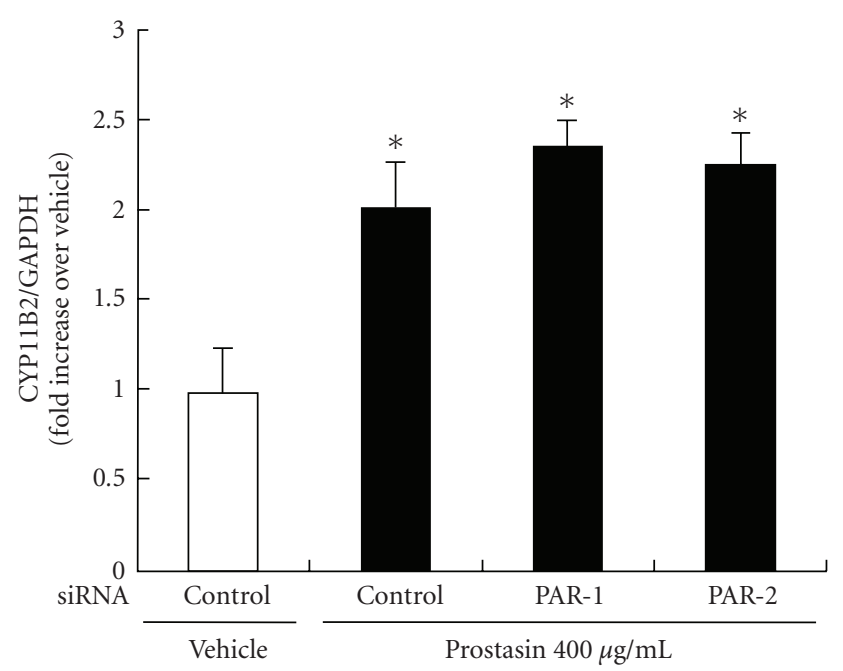

(c)

FIGURE 2: Effect of adenovirus-mediated overexpression of prostasin on CYP11B2 expression (a) and aldosterone production (b) in H295R cells. Cells were infected with $6 \times 10^{5} \mathrm{pfu}$ of control virus (Ad-LacZ) or adenovirus carrying human prostasin (Ad-hPRSS8) for 1 hour. Forty eight hours after infection, total RNA was isolated for the real time PCR and the medium was collected for the aldosterone measurement. (c) Effect of PAR-1 or PAR-2 knock down on prostasin-induced CYP11B2 expression in H295R cells. Cells were transfected with siRNA for either PAR-1 or PAR-2 for 48 hours and then treated with prostasin for another 24 hours. mRNA expression of CYP11B2 was determined by the real time PCR. Values are means $\pm \mathrm{SD}(n=6) .{ }^{*} P<.001$ versus Ad-LacZ (a), (b), or vehicle (c).

prostasin was blocked by the $\mathrm{Ca}^{2+}$ channel blocker and the PKC inhibitor. These results suggest that prostasin might play a regulatory role in the aldosterone production by adrenal grand.

Previously we reported that aldosterone increased prostasin abundance in rat and human [3]. Wang et al. demonstrated that adenovirus-mediated human prostasin gene delivery resulted in an increase in plasma prostasin and aldosterone levels as well as an elevation in blood pressure in rat [5]. These findings suggest the possibility that there may be a positive feedback regulation in the aldosterone production by prostasin. To elucidate the effect of the elevated circulating plasma prostasin levels on aldosterone production by adrenal gland, we generated recombinant prostasin protein and studied the effect of prostasin on aldosterone production in H295R cells. Previous studies in the early 1980s described the stimulatory effect of trypsin on aldosterone secretion from rat adrenal zona glomerulosa [11-13]. In addition, trypsin has been shown to activate PAR-1 and thereby stimulate aldosterone secretion from adrenal zona glomerulosa [14]. These studies suggest the possibility that trypsin-like serine proteases such as prostasin may be able to stimulate aldosterone production from adrenal gland. In the present studies, we demonstrated that treatment of $\mathrm{H} 295 \mathrm{R}$ cells with prostasin significantly increased both CYP11B2 mRNA expression and aldosterone production in a dose-dependent manner. Since the specific activity of our recombinant prostasin against synthetic substrate is approximately 30 -fold lower than the native prostasin purified from human seminal fluid (data not shown), the concentration of recombinant prostasin used for the current studies is relatively higher than the serum 


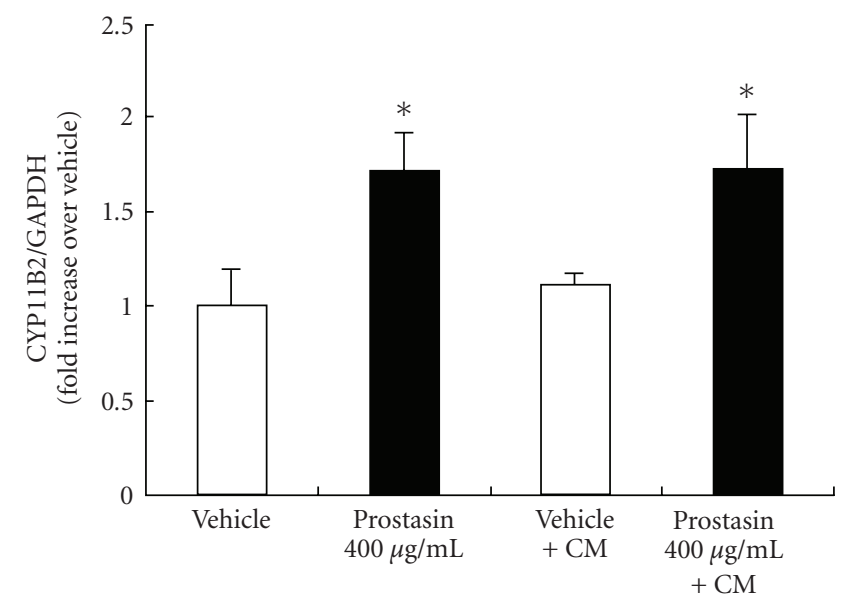

(a)

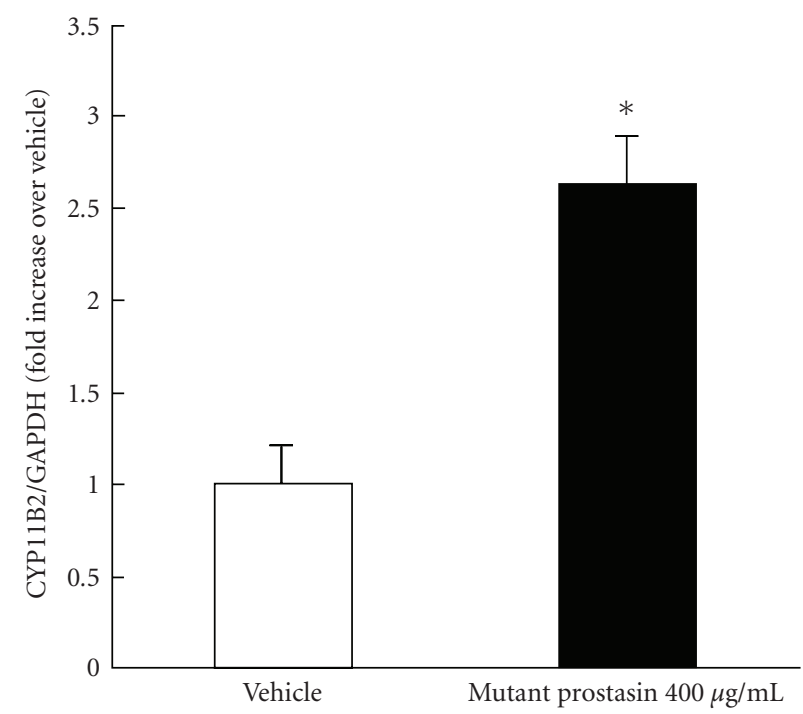

(c)

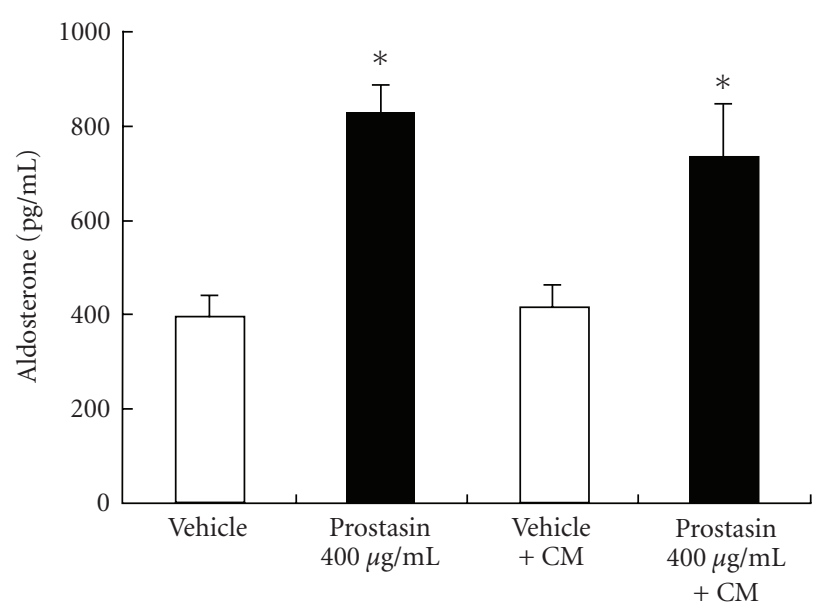

(b)

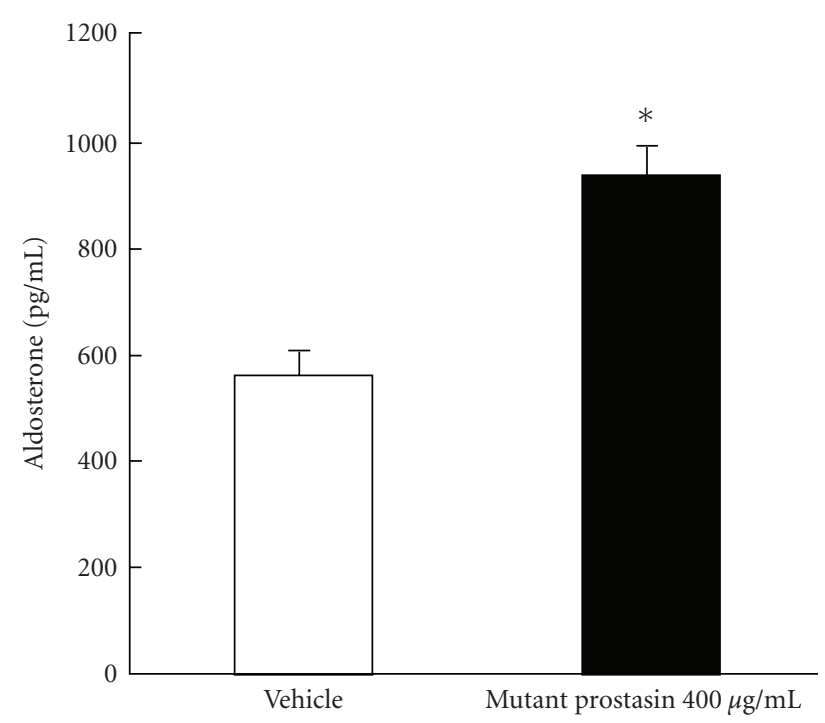

(d)

FIgURE 3: Prostasin increases CYP11B2 expression and aldosterone production independently of its proteolytic activity in H295R cells. (a) CYP11B2 expression was measured following 24-hour treatment with prostasin in the presence or absence of $100^{-} \mathrm{M}$ camostat mesilate (CM). (b) Aldosterone production was determined following 48-hour treatment with prostasin in the presence or absence of $100^{-} \mathrm{M}$ CM. (c) CYP11B2 expression was measured following 24-hour treatment with protease-dead mutant prostasin. (d) Aldosterone production was determined following 48 -hour treatment with mutant prostasin. Values are means $\pm \operatorname{SD}(n=6) .{ }^{*} P<.001$ versus vehicle.

prostasin concentration reported by Mok et al. However, contrary to our expectations, treatment with camostat mesilate, a potent prostasin inhibitor [15, 19], had no effect on the prostasin-induced aldosterone synthesis. In addition, the protease-dead mutant of prostasin significantly stimulated CYP11B2 expression and aldosterone production. These findings suggest that the protease activity is not primarily required for the prostasin-induced aldosterone synthesis in H295R cells. In the current studies, we used recombinant human prostasin generated by an insect expression system. We speculate that our recombinant protein may have different glycosylation modification or may contain misfolded proteins from the native prostasin due to the non-mammalian and forced expression system. As a result, our recombinant prostasin had lower specific activities, suggesting that our recombinant prostasin may have lower biological efficacy as well. Therefore, we think that our recombinant prostasin required relatively higher concentrations of proteins to observe the aldosterone synthesis in H295R cells. We also tested the effects of residue reagents to exclude the possibility that contaminant materials in the recombinant prostasin stimulated the aldosterone synthesis. We manipulated noninfected silkworm hemolymph exactly the same way as infected hemolymph and used as a control, but we did not observe any significant aldosterone synthesis in H295R cells (data not shown). Our findings that the gene silencing of either PAR-1 or PAR-2 had no effect on the prostasin-mediated increase in CYP11B2 expression may also support the nonproteolytic effect of prostasin. Because we were not able to demonstrate any 


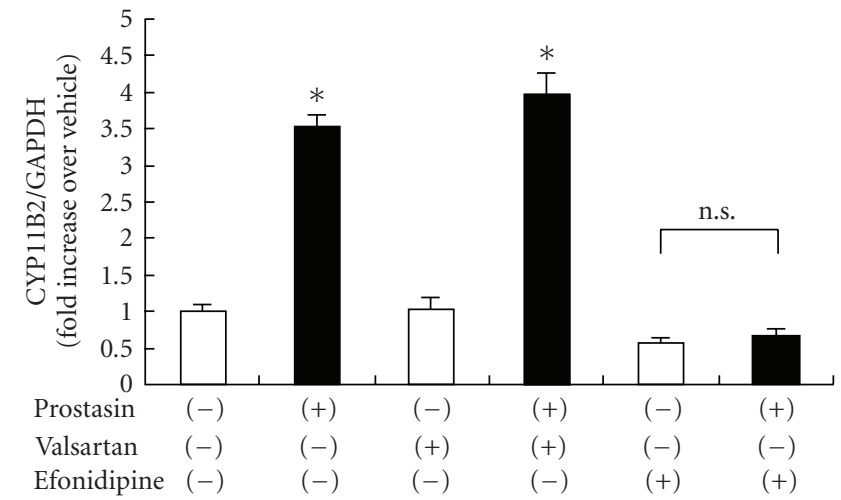

(a)

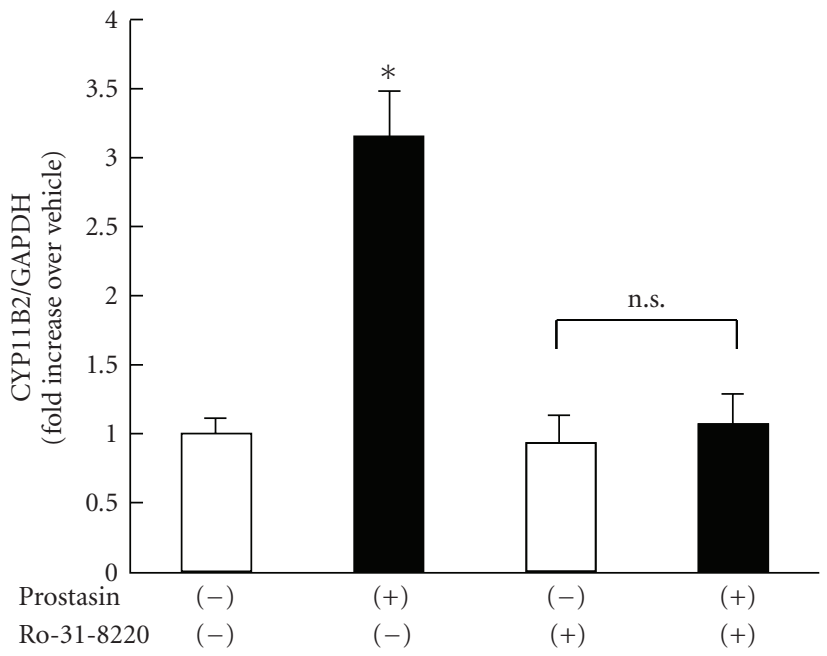

(c)

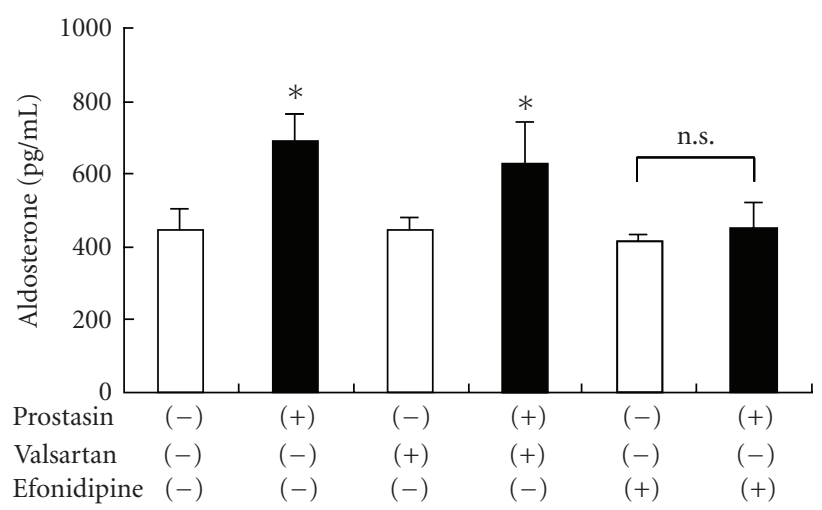

(b)

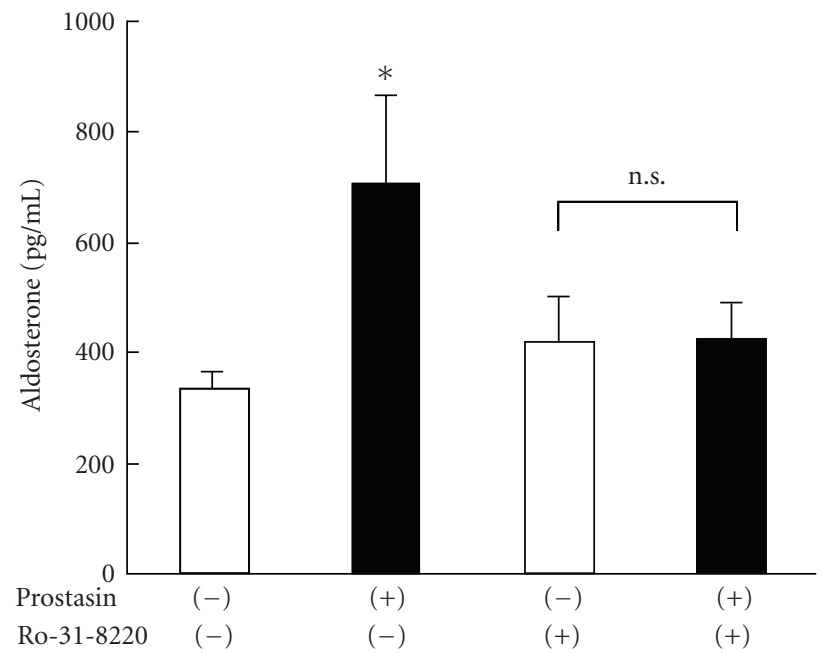

(d)

FIGURE 4: Effect of angiotensin II type 1 receptor blocker (a) and (b), T-type/L-type Ca ${ }^{2+}$ channel blocker (a) and (b), or PKCe inhibitor (c) and (d) on the prostasin-induced CYP11B2 expression and aldosterone production in H295R cells. Cells were treated with prostasin $(400 \mu \mathrm{g} / \mathrm{mL})$ in the presence or absence of valsartan $(100 \mathrm{nM})$, efonidipine (100 nM), or Ro-31-8220 ( $\left.1^{-} \mathrm{M}\right)$. CYP11B2 mRNA expression (a) and (c) was determined following 24-hour treatment, and aldosterone production (b) and (d) was evaluated following 48-hour treatment. Values are means $\pm \mathrm{SD}(n=6) .{ }^{*} P<.001$ versus vehicle. n.s.: not significant.

importance of the catalytic activity of prostasin on the aldosterone synthesis, we propose a direct protein-protein interaction between prostasin and unidentified membrane molecules such as transmembrane receptors. Noncatalytic biologic function in serine proteases has been illustrated by the hepatocyte growth factor and macrophage stimulatory protein hormones, both of which have lost their catalytic function but interact with transmembrane tyrosine kinase receptors to induce intercellular signaling cascades [20]. Also, Chen et al. reported the nonproteolytic function of prostasin in the regulation of mRNA expression of urokinase-type plasminogen activator (uPA) and inducible nitric oxide synthase (iNOS) in PC-3 human prostate carcinoma cells [21], although they did not elucidate the precise mechanism by which prostasin mediates its signal to the intracellular targets. Further investigations are definitely required to identify the target molecules for the extracellular prostasin to transmit its signal to the intracellular molecules leading to the activation of aldosterone synthesis.
Because the aldosterone synthesis is regulated by angiotensin II through the angiotensin II type 1 receptor (AT-1R) in normal human adrenocortical cells [22, 23], the first target molecule that we examined was AT-1R. We hypothesized that prostasin may transactivate the AT-1R in some way leading to the stimulation of CYP11B2 expression. However, since the treatment with valsartan did not abolish the aldosterone synthesis by prostasin, it is unlikely that angiotensin II signaling participates in this mechanism. Calcium influx through the plasma membrane has been shown to mediate the expression of mRNAs for CYP11B1 and CYP11B2, especially CYP11B2, in H295R cells. mRNA expression of these enzymes was suppressed by L-type $\mathrm{Ca}^{2+}$ channel blockers, indicating that L-type $\mathrm{Ca}^{2+}$ channels may be involved in the steroid biosynthesis in adrenocortical cells [24-26]. In addition, the earlier studies suggested the possible involvement of T-type $\mathrm{Ca}^{2+}$ channel activity in aldosterone biosynthesis in bovine adrenal glomerulosa cells [27]. Recently, efonidipine and benidipine, which are 
dual (T-type/L-type) $\mathrm{Ca}^{2+}$ channel blockers, have been demonstrated to inhibit aldosterone biosynthesis in H295R cells more potently than the L-type $\mathrm{Ca}^{2+}$ channel blocker nifedipine $[28,29]$. In the current studies, we showed that efonidipine, a T-type/L-type $\mathrm{Ca}^{2+}$ channel blocker, exerted an inhibitory effect on steroid biosynthesis by prostasin in H295R cells, suggesting the involvement of these channels in the aldosterone production by prostasin. Several PKC isozymes have been reported to mediate endogenous $\mathrm{PKC} \mu$ activation. Romero et al. showed that $\mathrm{PKC} \varepsilon$ has a critical role in $\mathrm{PKC}^{-}$activation in human adrenal cells and $\mathrm{PKC} \mu$ activation by angiotensin II caused a remarkable upregulation of aldosterone synthase gene expression in H295R cells [30]. They also showed that a PKCE inhibitor Ro-31-8220 inhibited the phosphorylation of $\mathrm{PKC} \mu$. We demonstrated that Ro-31-8220 significantly reduced the aldosterone synthesis by prostasin, suggesting the involvement of PKC $\varepsilon$ in the prostasin-mediated aldosterone synthesis pathway.

In conclusions, we demonstrated that prostasin increased aldosterone production in H295R cells, probably via nonproteolytic action of prostasin, and that T-type/L-type calcium channels and $\mathrm{PKC} \varepsilon$ are at least involved in the aldosterone synthesis by prostasin. Although the precise mechanism by which extracellular prostasin transmits its signal to the intracellular molecules remains unknown, our current data will provide a new insight into the role of prostasin, especially that in the systemic circulation, in the regulatory mechanisms of aldosterone production by the adrenal gland. Also, our current findings, together with our previous reports, suggest the possibility that there may be a vicious cycle between prostasin and aldosterone although further intensive in vivo studies are required to elucidate the physiological roles of prostasin in the systemic circulation.

\section{Acknowledgments}

This work was supported by the Grants-in-Aid for Scientific Research from the Ministry of Education, Culture, Sports, Science and Technology in Japan (19590956 to K. Kitamura, 19590958 to T. Miyoshi, 18790570 to N. Shiraishi, 18790569 to M. Adachi, and 18390252 to K. Tomita); Salt Science Research Foundation Grant (0728 to K. Kitamura); Mitsubishi Pharma Research Foundation Grant (to K. Kitamura); and Advanced Education Program for Integrated Clinical, Basic and Social Medicine, Kumamoto University (Support Program for Improving Graduate School Education, MEXT, Japan).

\section{References}

[1] J. X. Yu, L. Chao, and J. Chao, "Prostasin is a novel human serine proteinase from seminal fluid. Purification, tissue distribution, and localization in prostate gland," The Journal of Biological Chemistry, vol. 269, no. 29, pp. 18843-18848, 1994.

[2] J. X. Yu, L. Chao, and J. Chao, "Molecular cloning, tissue-specific expression, and cellular localization of human prostasin mRNA," The Journal of Biological Chemistry, vol. 270, no. 22, pp. 13483-13489, 1995.
[3] T. Narikiyo, K. Kitamura, M. Adachi, et al., "Regulation of prostasin by aldosterone in the kidney," Journal of Clinical Investigation, vol. 109, no. 3, pp. 401-408, 2002.

[4] A. Koda, N. Wakida, K. Toriyama, et al., "Urinary prostasin in humans: relationships among prostasin, aldosterone and epithelial sodium channel activity," Hypertension Research, vol. 32, pp. 276-281, 2009.

[5] C. Wang, J. Chao, and L. Chao, "Adenovirus-mediated human prostasin gene delivery is linked to increased aldosterone production and hypertension in rats," American Journal of Physiology, vol. 284, no. 4, pp. R1031-R1036, 2003.

[6] K. M. Curnow, M.-T. Tusie-Luna, L. Pascoe, et al., "The product of the CYP11B2 gene is required for aldosterone biosynthesis in the human adrenal cortex," Molecular Endocrinology, vol. 5, no. 10, pp. 1513-1522, 1991.

[7] L. J. Domalik, D. D. Chaplin, M. S. Kirkman, et al., "Different isozymes of mouse $11 \beta$-hydroxylase produce mineralocorticoids and glucocorticoids," Molecular Endocrinology, vol. 5, no. 12, pp. 1853-1861, 1991.

[8] T. Kawamoto, Y. Mitsuuchi, K. Toda, et al., "Role of steroid $11 \beta$-hydroxylase and steroid 18-hydroxylase in the biosynthesis of glucocorticoids and mineralocorticoids in humans," Proceedings of the National Academy of Sciences of the United States of America, vol. 89, no. 4, pp. 1458-1462, 1992.

[9] T. Ogishima, H. Suzuki, J.-I. Hata, F. Mitani, and Y. Ishimura, "Zone-specific expression of aldosterone synthase cytochrome $\mathrm{P}-450$ and cytochrome $\mathrm{P}-45011 \beta$ in rat adrenal cortex: histochemical basis for the functional zonation," Endocrinology, vol. 130, no. 5, pp. 2971-2977, 1992.

[10] M. H. Bassett, P. C. White, and W. E. Rainey, "The regulation of aldosterone synthase expression," Molecular and Cellular Endocrinology, vol. 217, no. 1-2, pp. 67-74, 2004.

[11] P. W. Raven, E. McCredie, G. P. Vinson, et al., "Effects of proteolytic enzymes on steroid release from rat adrenal zona glomerulosa tissue: evidence for novel steroid-protein complexes," Biochemical and Biophysical Research Communications, vol. 104, no. 4, pp. 1247-1254, 1982.

[12] P. W. Raven, E. McCredie, M. McAuley, and G. P. Vinson, "Origins of the differences in function of rat adrenal zona glomerulosa cells incubated as intact tissue and as collagenaseprepared cell suspensions," Cell Biochemistry and Function, vol. 1, no. 1, pp. 17-24, 1983.

[13] P. W. Raven, M. E. McAuley, and G. P. Vinson, "Serine proteases selectively control the output of 18hydroxycorticosterone and aldosterone in stimulated zona glomerulosa tissue of the rat adrenal," Journal of Endocrinology, vol. 99, no. 1, pp. 13-22, 1983.

[14] P. W. Raven, S. Kapas, M. Carroll, and J. P. Hinson, "Aldosterone secretion by the rat adrenal cortex is stimulated by the activation of protease-activated receptor 1," Journal of Endocrinology, vol. 169, no. 3, pp. 581-585, 2001.

[15] A. Maekawa, Y. Kakizoe, T. Miyoshi, et al., "Camostat mesilate inhibits prostasin activity and reduces blood pressure and renal injury in salt-sensitive hypertension," Journal of Hypertension, vol. 27, no. 1, pp. 181-189, 2009.

[16] M. H. Bassett, Y. Zhang, C. Clyne, P. C. White, and W. E. Rainey, "Differential regulation of aldosterone synthase and $11 \beta$-hydroxylase transcription by steroidogenic factor-1," Journal of Molecular Endocrinology, vol. 28, no. 2, pp. 125-135, 2002.

[17] Y. Kakizoe, K. Kitamura, T. Ko, et al., "Aberrant ENaC activation in Dahl salt-sensitive rats," Journal of Hypertension, vol. 27, no. 8, pp. 1679-1689, 2009. 
[18] G. P. Vinson, S. M. Laird, J. P. Hinson, N. Mallick, S. Marsigliante, and R. Teja, "Trypsin stimulation of aldosterone and 18-hydroxycorticosterone production by rat adrenal zona glomerulosa tissue is mediated by activation of protein kinase C," Journal of Molecular Endocrinology, vol. 5, no. 1, pp. 85-93, 1990.

[19] K. Coote, H. C. Atherton-Watson, R. Sugar, et al., "Camostat attenuates airway epithelial sodium channel function in vivo through the inhibition of a channel-activating protease," Journal of Pharmacology and Experimental Therapeutics, vol. 329, no. 2, pp. 764-774, 2009.

[20] K. A. Stuart, S. M. Riordan, S. Lidder, L. Crostella, R. Williams, and G. G. Skouteris, "Hepatocyte growth factor/scatter factorinduced intracellular signalling," International Journal of Experimental Pathology, vol. 81, no. 1, pp. 17-30, 2000.

[21] M. Chen, Y.-Y. Fu, C.-Y. Lin, L.-M. Chen, and K. X. Chai, "Prostasin induces protease-dependent and independent molecular changes in the human prostate carcinoma cell line PC-3," Biochimica et Biophysica Acta, vol. 1773, no. 7, pp. 1133-1140, 2007.

[22] I. M. Bird, N. A. Hanley, R. A. Word, et al., "Human NCIH295 adrenocortical carcinoma cells: a model for angiotensinII-responsive aldosterone secretion," Endocrinology, vol. 133, no. 4, pp. 1555-1561, 1993.

[23] I. M. Bird, J. I. Mason, and W. E. Rainey, "Hormonal regulation of angiotensin II type 1 receptor expression and AT1-R mRNA levels in human adrenocortical cells," Endocrine Research, vol. 21, no. 1-2, pp. 169-182, 1995.

[24] I. M. Bird, J. M. Mathis, J. I. Mason, and W. E. Rainey, "Ca ${ }^{2+}$ regulated expression of steroid hydroxylases in H295R human adrenocortical cells," Endocrinology, vol. 136, no. 12, pp. 56775684, 1995.

[25] C. D. Clyne, P. C. White, and W. E. Rainey, "Calcium regulates human CYP11B2 transcription," Endocrine Research, vol. 22, no. 4, pp. 485-492, 1996.

[26] K. Denner, W. E. Rainey, V. Pezzi, I. M. Bird, R. Bernhardt, and J. M. Mathis, "Differential regulation of $11 \beta$-hydroxylase and aldosterone synthase in human adrenocortical H295R cells," Molecular and Cellular Endocrinology, vol. 121, no. 1, pp. 8791, 1996.

[27] M. F. Rossier, E. A. Ertel, M. B. Vallotton, and A. M. Capponi, "Inhibitory action of mibefradil on calcium signaling and aldosterone synthesis in bovine adrenal glomerulosa cells," Journal of Pharmacology and Experimental Therapeutics, vol. 287, no. 3, pp. 824-831, 1998.

[28] O. Akizuki, A. Inayoshi, T. Kitayama, et al., "Blockade of T-type voltage-dependent $\mathrm{Ca}^{2+}$ channels by benidipine, a dihydropyridine calcium channel blocker, inhibits aldosterone production in human adrenocortical cell line NCI-H295R," European Journal of Pharmacology, vol. 584, no. 2-3, pp. 424434, 2008.

[29] K. Imagawa, S. Okayama, M. Takaoka, et al., "Inhibitory effect of efonidipine on aldosterone synthesis and secretion in human adrenocarcinoma (H295R) cells," Journal of Cardiovascular Pharmacology, vol. 47, no. 1, pp. 133-138, 2006.

[30] D. G. Romero, B. L. Welsh, E. P. Gomez-Sanchez, L. L. Yanes, S. Rilli, and C. E. Gomez-Sanchez, "Angiotensin IImediated protein kinase D activation stimulates aldosterone and cortisol secretion in H295R human adrenocortical cells," Endocrinology, vol. 147, no. 12, pp. 6046-6055, 2006. 

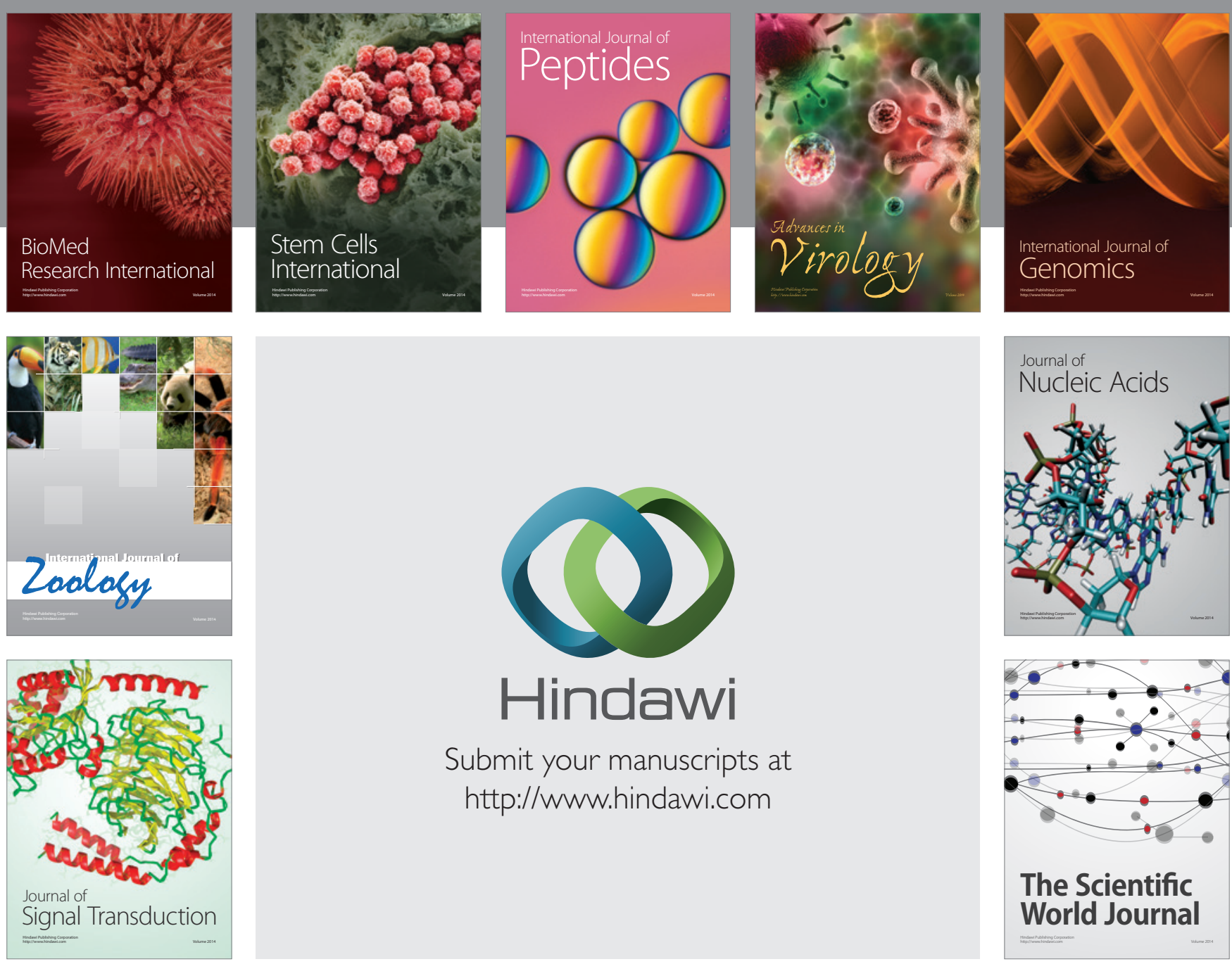

Submit your manuscripts at

http://www.hindawi.com
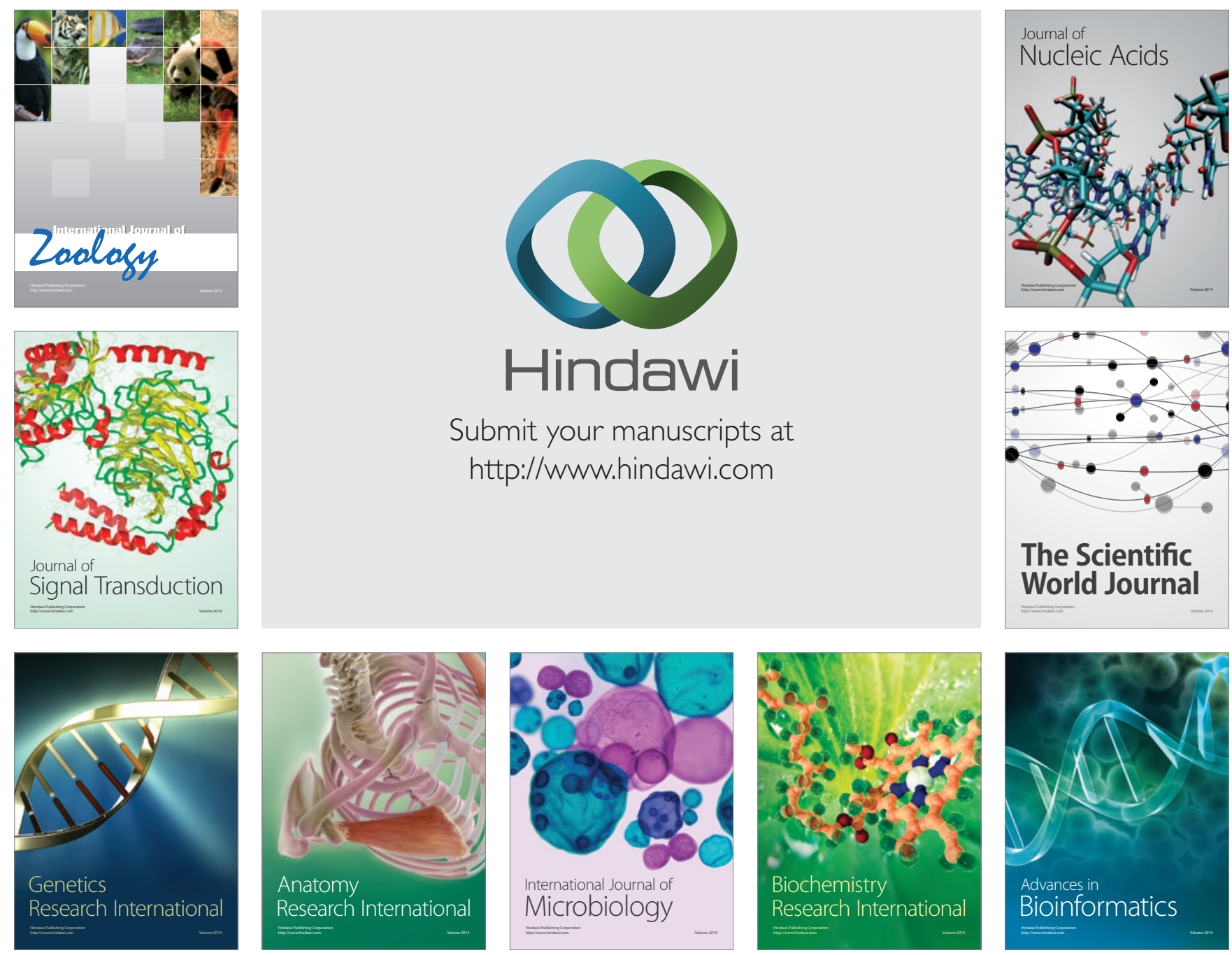

The Scientific World Journal
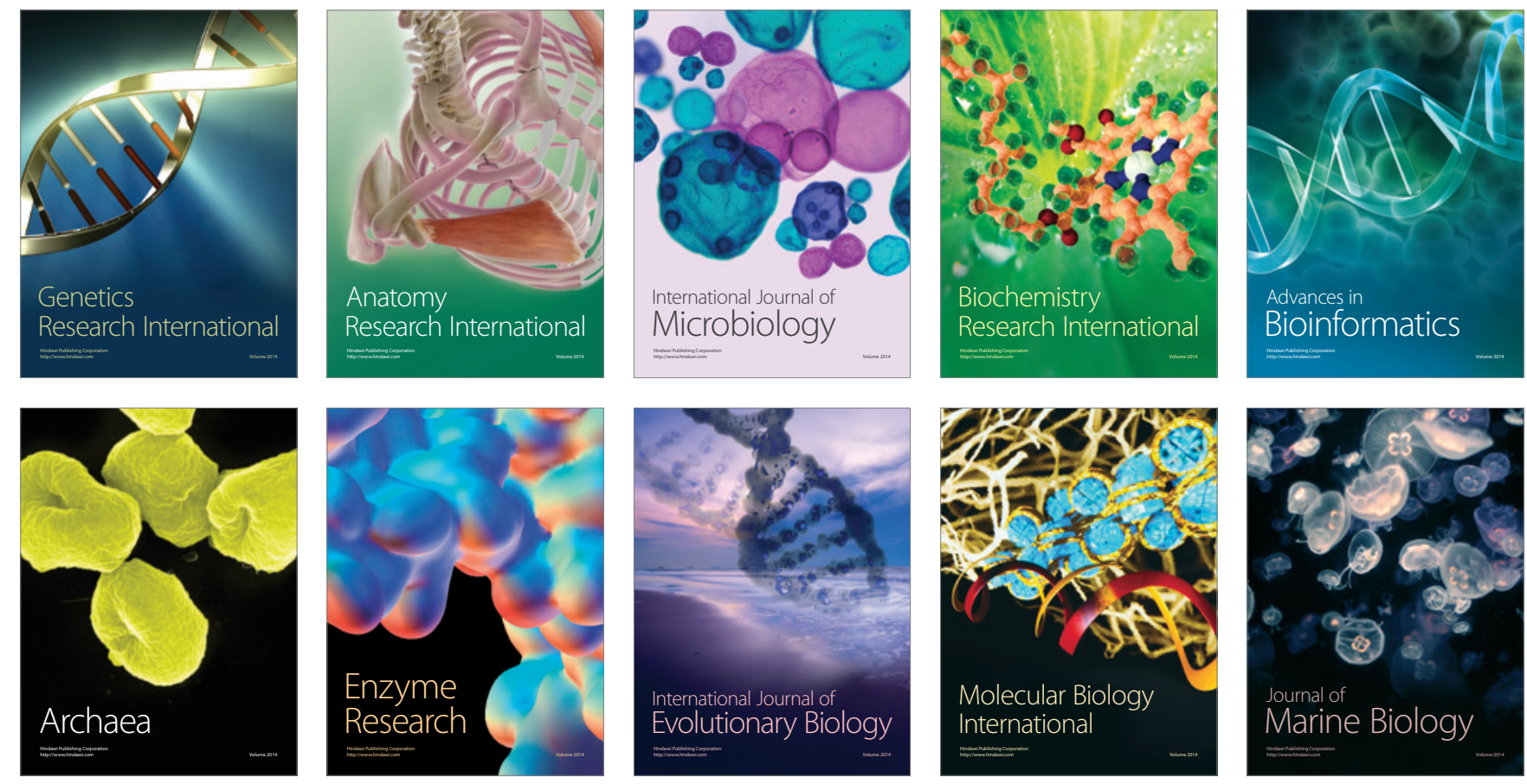